\title{
Combination of Atomic Force Microscopy and Principal Component Analysis As a General Method for Direct Recognition of Functional and Structural Domains in Nanonocomposite Materials
}

\author{
BRUNO TORRE ${ }^{1 *}$ MANUELE BICEGO,${ }^{1,2}$ MARCO CRISTIANI, ${ }^{1,2}$ VITTORIO MURINO,${ }^{1,2}$ ALBERTO DIASPRO, ${ }^{1}$ \\ AND ROBERTO CINGOLANI ${ }^{1}$ \\ ${ }^{1}$ Istituto Italiano di Tecnologia (IIT). Via Morego 30, Genova 16163, Italy \\ ${ }^{2}$ Dipartimento di Informatica Università degli Studi di Verona, Ca' Vignal 2, Strada Le Grazie 15, Verona 37134, Italy
}

KEY WORDS nanotechnology; microscopy; functional materials; functional recognition; dynamic spectroscopy; dimensionality reduction; principal component analysis

\begin{abstract}
In this work, we report a simple method to direct identify nanometer sized textures in composite materials by means of AFM spectroscopy, aiming at recognizing structured region to be further investigated. It consists in acquiring a set of dynamic data organized in spectroscopy maps and subsequently extracting most valuable information by means of the principal component analysis (PCA) method. This algorithm projects the information of $D$ spectroscopy curves, each containing $P$ data, acquired at each point of an $L x C$ grid into a subset of $L x C$ maps without any assumption on the sample structure, filtering out redundancies and noise. As a consequence, a huge amount of $3 \mathrm{D}$ data is condensed into few $2 \mathrm{D}$ maps, easy to be examined. Results of this algorithm allow to find and locate regions of interest within the map, allowing a further reduction of data series to be extensively analyzed or modeled. In this work, we explain the main features of the method and show its application on a nanocomposite sample. Microsc. Res. Tech. 73:973-981, 2010. ๑ 2010 Wiley-Liss, Inc.
\end{abstract}

\section{INTRODUCTION}

Local properties of materials with nanometer resolution can be probed by means of atomic force microscope, performing force spectroscopy experiments: in these experiments, tip-sample interaction forces are measured acquiring quasi-static cantilever deflection as a function of separation while the tip is brought into contact with the sample and then far apart from it. Force-distance (FD) curves contain valuable information about nanoscale material properties such as adhesion, elasticity and plasticity, as well as friction (Cappella and Dietler, 1999).

Interaction depends both on sensor (cantilever and tip) and on the specimen properties: calibration of the first and a proper modeling of the interaction allow reconstructing local feature of the sample. Resolution of the order of contact area, usually $10 \mathrm{~nm}$ or better, can be obtained.

The choice of the proper mathematical model requires a priori knowledge of the interaction and is affected by experimental uncertainties (e.g., tip curvature radius): commonly used mathematical models, as Hertz (Herz, 1882), JKR (Johnson et al., 1971) and DMT (Derjaguinet al., 1975) models, take into account rounded tips with well known curvature radius and flat surface-hypothesis is not always verified-in presence (JKR and DMT) or in absence (Hertz) of adhesion. In many interesting case -such as biological samples and nanocomposites-a more refined model is often needed, also including additional parameters (such as roughness or specific binding): as conse- quence, computational weight for modeling rapidly increase; an accurate evaluation of each FD curve of a $3 \mathrm{D}$ spectroscopy map (2D arrays of FD curves) is, in most cases, time consuming; therefore information in them is often retrieved selecting few representative curves and considering few features in them.

If the same operation is done while dithering the cantilever close to its resonance frequency, tip-sample interaction is probed in dynamic mode (dynamic force spectroscopy): several parameters can be measured as a function of distance (such as static deflection, amplitude, phase, higher harmonics, frequency, etc.) containing a larger amount of data: valuable information about local interaction can be extracted or reconstructed, revealing material properties such as short and long ranges forces (Giessibl, 1997), friction (Holscher et al.,1998), plasticity (Butt et al., 2005), chemical composition (Magonov et al.,1997) and so on. Dynamic parameters have been first inverted to reconstruct tip-sample interaction forces in case FM-AFM by Durig using Hamilton-Jacobi perturbation theory in large amplitude oscillation-or short range forcescase (Durig, 2000), and then generalized by Sader et al. (2004, 2005) considering first resonance. Durig also investigated dynamic behavior by considering ampli-

\footnotetext{
*Correspondence to: Dr. Bruno Torre, Istituto Italiano di Tecnologia (IIT)., Via Morego, 3016163 Genova, Italy. E-mail: bruno.torre@iit.it

Received 15 January 2010; accepted in revised form 18 January 2010 DOI 10.1002/jemt.20837

Published online 15 March 2010 in Wiley Online Library (wileyonlinelibrary.com).
} 


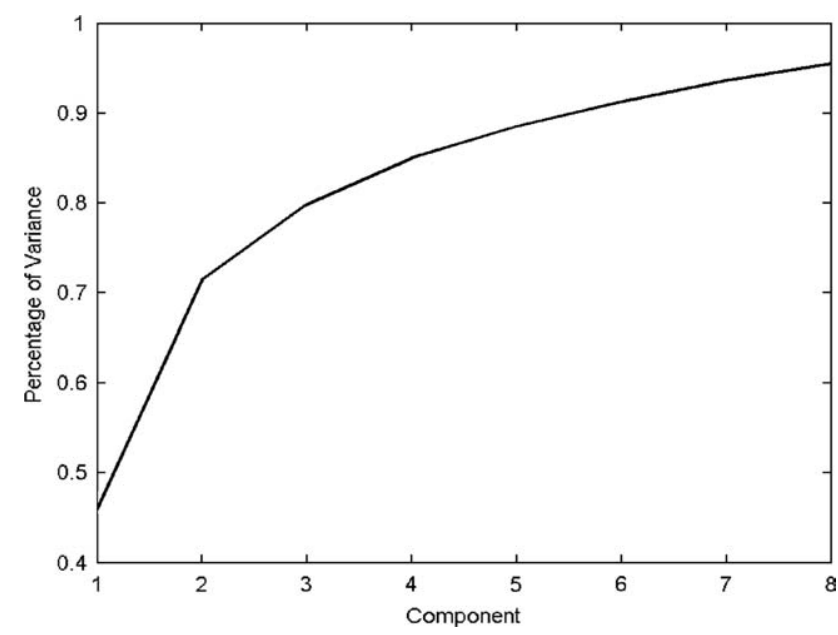

Fig. 1. Convergence of variance as a function of components number in PCA. Depending on the signal, number of component to reach the threshold of 95 or $98 \%$ of variance can span from some units to some or several tens. Most relevant part of the information is attributed to these components, while others are regarded as noise.

tude and phase on higher harmonics, using Chebyshev polynomial expansion method (Durig, 1999).

In AM-AFM case Holscher (2006) developed an integral equation allowing the inversion of experimental parameters in case short range (large amplitude) interactions. More general case has been considered by Lee and Jhe (2006) using modified Bessel functions and by $\mathrm{Hu}$ and Raman (2008) using Chebyshev polynomial expansion method. Sugawara proposed that phaseModulation method allows preventing tip phase jump instabilities (Sugawara et al., 2007); this has been experimentally verified by Holscher (2008) using non commercial hardware setup.

Under particular oscillating regimes, also subharmonic and chaotic cantilever dynamics, fingerprint of tip-sample interactions, have been found (Jamitzky et al., 2006).

All these huge amount of information requires a higher computational weight to reconstruct physically valuable parameters from comparison with contact models (Unertl, 1999); as a result a fast and easy analysis relying on these dynamic methods is still far to be routinely implemented to spectroscopy maps or it is limited to few information.

Recently, principal component analysis (PCA) method has been successfully applied to compress complex data series from band excitation (BE) technique (Jesse et al., 2007; Jesse and Kalinin, 2009) to highlight contrast on magnetic and piezoelectric structure, for further modeling and analysis.

In this work, we focus on cantilever kinematics, as an intermediate step to facilitate further analysis, in order to classify dynamic spectroscopy maps on the basis of a reduced parameter set, using a widely applicable statistical analysis. It consists in applying PCA algorithm on a $L x C$ ( $L$ lines by $C$ columns) spectroscopy map, where each voxel is a set of $2 x D$ arrays of $P$ points (usually several hundred to some thousands) from independently sampling $D$ parameters (e.g., amplitude and phase on fundamental and higher harmonics,
TABLE 1. Most relevant cantilever and tip parameters for the sensor selected to collect data presented below

\begin{tabular}{lcr}
\hline \multicolumn{1}{c}{ Parameter } & Nominal value & Measured value \\
\hline Shape & Rectangular & \\
Dimensions $\mathrm{L} \times \mathrm{W} \times \mathrm{H}(\mu \mathrm{m})$ & $240 \times 30 \times 2.8$ & \\
Spring constant $(\mathrm{N} / \mathrm{m})$ & 2 & 2.1 \\
Resonance frequency $(\mathrm{kHz})$ & 70 & 74.478 \\
Q factor & & 195 \\
Inverse amplitude optical & & \\
$\quad$ lever sensitivity $(\mathrm{nm} / \mathrm{V})$ & 14 & \\
Tip height $(\mu \mathrm{m})$ & $<7$ & \\
Tip curvature radius & & \\
\hline
\end{tabular}

Values in column two are calculated from geometric nominal parameters, while corresponding positions in column three are measured using thermal noise excitation method.

deflection, frequency, damping etc.) while approaching and retracting from the surface. At a first step, data is analyzed separately on each channel, grouped in $D$ maps containing $L x C x P x 2$; subsequently data from all channels (with the exception of Z LVDT, see below) is grouped together and analyzed: in this way data is reduced to $D+1$ maps with $L x C$ dimensionality, summing up independent information from each parameter and from all of them. This two-step process is intended to find different features within the probed region and to evaluate, at the same time, the response on different dynamic parameters: results therefore provide a robust and quick screening method to locate region of interest (ROI) on the sample, where further investigations can be addressed, also providing indications on the type of analysis that can be used to highlight features.

\section{MATERIALS AND METHODS Sample Preparation}

Sample is prepared using a $1 \mathrm{mM}$ solution of $99 \% \mathrm{wt}$ of poly(ethylmethacrylate-comethylacrylate) (PEMMA) and $1 \%$ wt. of $\gamma-\mathrm{Fe}_{2} \mathrm{O}_{3}$ nanoparticles of in chloroform as described in Fragouli et al. (in preparation); a film obtained drop casting the solution on a clean glass and drying it in saturated chloroform environment overnight without external magnetic film. Film is embedded in EPON $^{\mathrm{TM}} 812$ resin and $200-\mathrm{nm}$ thick slices are cut using a Leica EM UC6 Ultramicrotome, with cut direction normal to the polymer film, so that each slice expose a sharp polymer-resin interface. Slices have been then suspended in de-ionized water and deposited on a microscope glass substrate. A sharp buried interface between polymer and resin is accessible for AFM measurements. TEM images on sample series prepared from the same film (data not shown) reveal 100-200$\mathrm{nm}$ thick particle layer at the interface, corresponding to a uniform coverage on the polymer film at top surface.

\section{AFM Measurements}

Data is acquired by means of MFP-3D atomic force microscope (Asylum Research, Santa Barbara CA) using Olympus OMCL-AC240TS rectangular silicon cantilevers (sensor parameters can be find in Table 1). These sensors have a intermediate spring constant between contact and noncontact cantilevers, can therefore be used for spectroscopy and imaging purpose both in static and dynamic mode: many metal coated canti- 
levers for special applications (e.g., magnetic or conductive coverage on the tip) can be find in this stiffness range, since for many application both contact and non contact investigation can be useful. More, a first resonance around $70 \mathrm{kHz}$ allows investigations both at higher cantilever resonances and harmonics within the detection bandwidth of almost all commercial microscope, useful to investigate mechanical properties.

Amplitude inverse optical lever sensitivity and other cantilever parameters in column three have been calibrated performing deflection measurements by force distance curve on a rigid glass substrate and thermal noise calibration method.

All measurements have been performed dithering the cantilever with a free oscillation amplitude of $260 \mathrm{~nm}$ at excitation frequency of $-73 \mathrm{~Hz}$ with respect to its resonance. Imaging have been performed in AM mode using an amplitude setpoint of $72 \%$ of the free oscillation, corresponding to oscillation amplitude of $\sim 185 \mathrm{~nm}$ and to a maximum withdrawing force of almost $400 \mathrm{nN}$.

Spectroscopy series is a curve collection on a $64 \times 64$ grid on a $5 \times 5 \mu^{2}$ region of the slice, crossing the interface between polymer and resin. Data from four different channels is acquired during approach and retract procedures, obtaining eight spectroscopy curves in each point of the grid. In our case we have:

1. Amplitude: oscillation amplitude synchronous with excitation, this is the channel used for the feedback in AM-mode; it is used as trigger channel to define the end point of each approach curve (and starting point of retraction):

2. Amplitude2: it is the amplitude at a second frequency on the deflection signal, monitored during experiment. It can be tuned to monitor cantilever behavior at frequencies different from fundamental one. Interesting processes can be higher harmonics of excitation signal, to monitor harmonic distortion or higher cantilever resonant modes; since higher harmonics depend on elastic properties of the sample (Durig, 2000), can be monitored to see changes in material composition. In this work we measure second harmonic distortion.

3. Phase: the phase lag of the cantilever oscillation with respect to excitation signal. This parameter is affected by dissipative tip-sample interactions (Garcia and Perez, 2002);

4. Frequency: this channel tracks frequency shift due to attractive and repulsive regime. Frequency shift depend on the mean force derivative, averaged over one oscillation period, therefore is sensitive changes between attractive and repulsive regime: attractive forces generate a frequency shift toward lower frequencies, while repulsive ones have the opposite effect.

More channels can be added using external data acquisition systems.

In addition, signal from positioning sensor (Z LVDT) on the vertical axis is taken, providing an absolute measurement of the $\mathrm{Z}$ position at each spectroscopy point. As approach and retract cycle is performed at constant speed, time dependence of this signal is a
TABLE 2. Acquisition parameters used during spectroscopy experiment

\begin{tabular}{lr}
\hline Spectroscopy parameter & Value \\
\hline Approach Speed $(\mu \mathrm{m} / \mathrm{s})$ & 1 \\
Retract Speed $(\mu \mathrm{m} / \mathrm{s})$ & 1 \\
Dwell time & 0 \\
Sampling frequency $(\mathrm{Hz})$ & 2,147 \\
Start distance, above surface $(\mathrm{nm})$ & 700 \\
\hline
\end{tabular}

For each approach and retract cycle, time dependence of vertical position $Z(t)$ (monitored on first channel) is a triangular curve with symmetrical slopes. Position of the cusp reveals height at the fixed trigger point, therefore contains morphology information.

triangular wave, whose cusp fall at the trigger point of spectroscopy data. This is the only channel that is not fed to PCA algorithm, but used independently to extract additional topography information, filtered out elsewhere.

Acquisition parameters used in this work are listed in Table 2.

Using before mentioned settings, a $64 \times 64$ map takes $\sim 2 \mathrm{~h}$ to be performed, with time series depth of more than 2,000 data for each parameter. This time can be remarkably lowered by reducing the number of point or by small increase of the measurement speed: for instance a $32 \times 32$ map with $2 \mu \mathrm{m} / \mathrm{s}$ takes less than $20 \mathrm{~min}$, making the time window suitable even for experiments on living cells or on most unstable samples.

Data set for each map is often of the order of hundreds of megabyte, and needs to be reduced to be analyzed. Following paragraph explains the mathematical tool used to reduce information with limited data loss.

\section{METHODOLOGY}

The presented methodology is aimed at pursuing the following objective: reduce the time series to a more manageable low dimensionality vector, possibly integrating the contribution of different channels.

In more details, the goal is to consider the whole set of time series, with different channels, and to reduce the dimensionality to a more manageable entity.

Before describing in detail the applied methodology, let us briefly summarize the theory and the practical aspects underlying the main technique, namely the PCA (Jollife, 1986). The code employed in the analysis has been written in MATLAB; in particular, the routine "pca" has been used, that comes from the PRTOOLS toolbox (downloadable from http://www. prtools.org/)

\section{Principal Components Analysis}

PCA aims at reducing the $N$-dimensional vector $\bar{x}$ which contains the original data to a compressed vector $\bar{c}$ which is $M$-dimensional, where $M<N$.

This resembles the encoder/decoder problem, depicted in the following diagram. A vector $\bar{x}$ is coded into a vector $\bar{c}$ with a reduced dimension. Vector $\bar{c}$ is then stored, transmitted or processed, which results in vector $\bar{c}^{\prime}$, which can be decoded back to a vector $\stackrel{\widetilde{x}^{\prime}}{\text {. This }}$ last vector is an approximation of the result which would have been attained by storing, transmitting or processing vector $\bar{x}$.

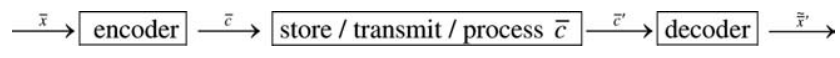


The encoder in this diagram should perform a linear operation, using a matrix $\overline{\bar{Q}}: \bar{c}=\overline{\bar{Q}} \bar{x}$

The decoder is also a linear operation, which can be written as a sum of the vector elements of $\bar{c}$ multiplied by the columns of matrix $\overline{\bar{Q}}: \tilde{\bar{x}}=\bar{c}^{T} \overline{\bar{Q}}^{T} \rightarrow \widetilde{\bar{x}}=\sum_{i=1}^{M} c_{i} \bar{q}_{i}$

PCA finds a linear operation so that the difference between $\bar{x}$ and $\bar{x}$ is minimal, attaining a considerable reduction of $\bar{x}$ when mapped into $\bar{c}$, i.e., minimizing the error between $\bar{x}$ and $\bar{x}$ :

$$
\begin{aligned}
\min E\left[\|\bar{x}-\tilde{\bar{x}}\|^{2}\right]=\min E\left[\sum_{i=1}^{N}\left(x_{i}-\tilde{x}_{i}\right)^{2}\right] \\
=\min E\left[\sum_{i=1}^{N}\left(x_{i}-\left(\sum_{j=1}^{N} c_{j} \bar{q}_{j}\right)_{i}\right)^{2}\right]
\end{aligned}
$$

The theory underlying PCA shows that the best data to omit from data vector $\bar{x}$ with a given correlation matrix $R$, is the data with the smallest variance. This is the data determined by the smallest eigenvalues of the matrix $R$.

Therefore, PCA starts by calculating the $N \times N$ correlation matrix of the data, thus estimating the correlation existent among the $N$ features of the sample set.

A correlation matrix $\bar{R}$ of a random vector $\bar{x}$ is the expectation of the outer product of the vector $\bar{x}$ with itself: $\overline{\bar{R}}=E\left[\bar{x}^{T} \bar{x}\right]$. In other words, $\overline{\bar{R}}$ is the covariance matrix of a pool of data samples whose features have been normalized with respect to their variances. In order to obtain a correlation matrix $\bar{R}$, we construct one by means of observations of different input vectors. We examine for example $K$ different images for constructing matrix $\bar{R}$ for a PCA of images. We note $\bar{x}^{(k)}$ as being the $k$-th observed vector.

We use the following empirical approximation of $\overline{\bar{R}}: \widetilde{R}_{i j}=\frac{1}{K} \sum_{k=1}^{K} x_{i}^{(k)} x_{j}^{(k)}$

The more observations are made, the better the approximation $\widetilde{\overline{\bar{R}}}$ of $\overline{\bar{R}}$ gets. Instead of matrix $\overline{\bar{R}}$ we use matrix $\widetilde{\bar{R}}$ in the PCA calculations.

\section{PCA in Four Steps}

The PCA can then be applied to all the vectors of the dataset: given a single vector $\bar{x}$ of dimension $N$ and the dataset correlation matrix $\overline{\bar{R}}$ we can reduce its dimension to $M$ (with $M<N$ ) in four steps:

1. Find the eigenvectors $\overline{\bar{Q}}$ and eigenvalues $\lambda_{i}$ of correlation matrix $\stackrel{\bar{R}}{R}: \overline{\bar{R}} \bar{q}_{i}=\lambda_{i} \bar{q}_{i}$

2. Arrange the eigenvalues in decreasing order: $\lambda_{1}>\lambda_{2}>. .>\lambda_{M}>. .>\lambda_{N}$

3. Pick up the eigenvectors which belong to the first $M$ largest eigenvalues.

4. The final reduced vector $\bar{c}$ is obtained by $c_{i}=\bar{x}^{T} \bar{q}_{i}$ for $i=1, . ., M$
In particular, the measure

$$
v_{\mathrm{tot}}=\frac{\sum_{1}^{M} \lambda_{i}}{\sum_{1}^{N} \lambda_{i}}
$$

represents the cumulative normalized variance, i.e., the total amount of variance captured by the first ordered $M$ eigenvectors.

At this point, it is important to note the underlying assumptions considered by PCA:

1. The data is assumed as organized as a $N$-dimensional Gaussian blob; this is also called the "Gaussian assumption." The calculation of the eigenvectors permits to rotate and scale opportunely the data, in order to align the blob with respect to its direction of maximal variance.

2 . In the novel, rotated and scaled coordinate system, each coordinate is assumed to be independent from each other. This means that PCA uses the eigenvectors of the correlation matrix and it only finds the independent axes of the data under the Gaussian assumption. For non-Gaussian or multi-modal Gaussian data, PCA simply de-correlates the axes.

3. PCA simply performs a coordinate rotation that aligns the transformed axes with the directions of maximum variance. It is only when we believe that the observed data has a high signal-to-noise ratio that the principal components with larger variance correspond to interesting dynamics and lower ones correspond to noise.

\section{DIMENSIONALITY REDUCTION}

In this part, the different steps of the proposed methodology are detailed. The idea is to preprocess the time series in order to align them starting from the trigger point at the end of approach (and beginning of retraction) of the tip on the surface. Stating from that point vectors are truncated to a fixed length containing all the valuable information in the interaction; this, on one side, eliminates all data far from the surface, where information is thought to be negligible, reducing vector representation (time series may be of different length due to surface roughness); on the other side, this procedure filters out topography information from all the channels by pairing all the curves by the same trigger point: this is intended to split topography and compositional information, to feed only the second one to the algorithm. Once given the fixed length vectorial representation, the PCA may be applied to eliminate the redundancy and to reduce the dimensionality to a more manageable entity. A further step, aimed at aggregating different channels information, may be inserted before the PCA computation.

\section{Step 1: Preprocessing}

In this step, the goal is to align the time series and to reduce them to a fixed length representation. The most reasonable way is to align them with respect to the contact point (which is stored in our experiments), retaining only a fixed amount of points before (approach curve) and after (retract curve) that point. Clearly, 


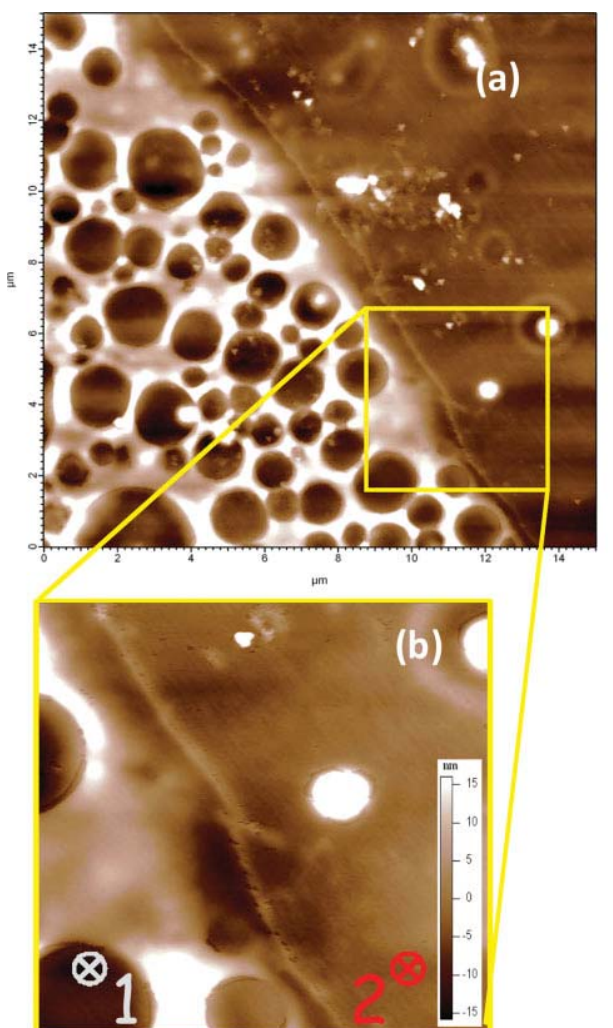

Fig. 2. Interface between EPON resin (gray mark) and PEMMA polymer (red mark). Image is acquired is amplitude modulation mode (excitation frequency-5\% of resonance frequency, setpoint $72 \%$ of free oscillation amplitude). Panel (b) shows the $5 \times 5 \mathrm{~mm}^{2}$ ROI selected from bigger $15 \times 15 \mu^{2}$ image $(\mathbf{a})$. Panels $(\mathbf{c}-\mathbf{f})$ show spec-

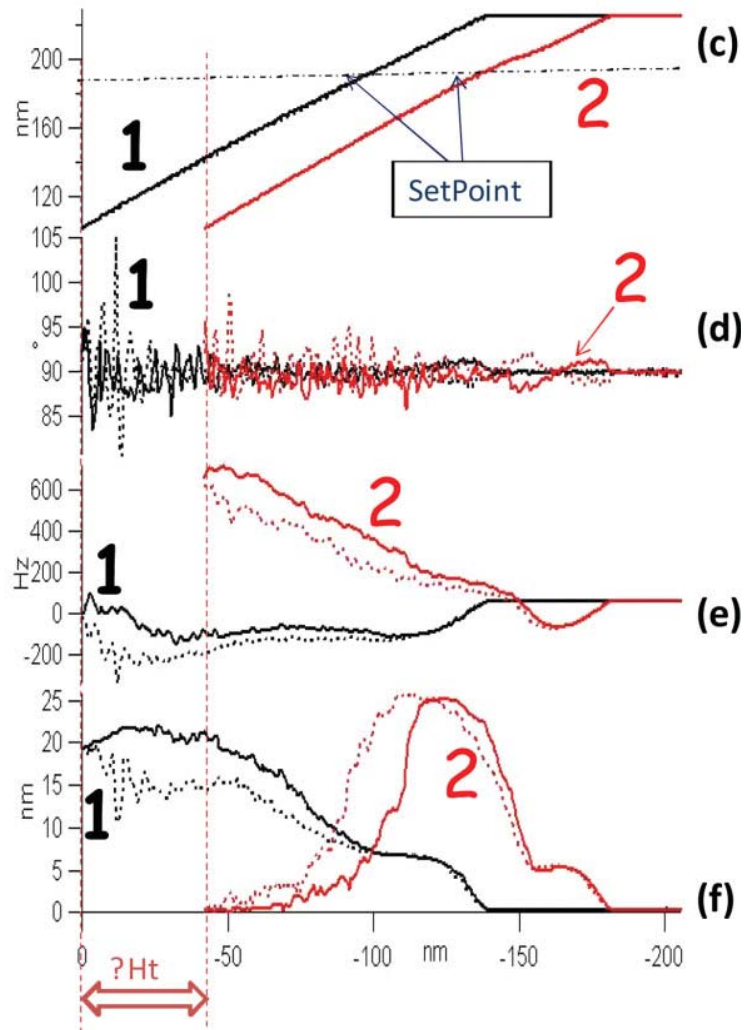

troscopy data taken in positions 1 and 2: channels acquired are Amplitude (c), Phase (d) frequency (e) and Amplitude 2 at $\left(f_{2}=2 \times f_{\text {exc }}\right)$; continuous and dotted lines are approach and retract curves, respectively. [Color figure can be viewed in the online issue, which is available at wileyonlinelibrary.com.] points far away from the contact point are less important, so that can be disregarded in the analysis. In all our experiments, we considered 250 points before and 250 after the contact point.

\section{Step 2: Integration of Different Channels}

Given the flexibility of the PCA, which is requiring in input only a generic vector of data, channel integration may be easily obtained by simply concatenating the time series relative to each channel (after the preprocessing). Actually PCA does not consider the order of the elements, but just the single contributions: in this way the convenient concatenation permit to have a single compact representation containing all the information obtainable from the different channels.

\section{Step 3: Data Reduction}

Given the input vectors, PCA is applied as described in the previous section. Choosing the right number of components to be retained (namely the dimension $M$ of the resulting vectors) may be somehow problematic, if the resulting representation should be used to perform a further analysis. A classical solution suggests retaining a number of components sufficient to explain a given amount of variance (like $95 \%$ or $98 \%$ ).

In Figure 1 there is an example concerning the typical result of the application of this technique to AFM data (see the experimental part for more details). In particular, the plot shows the amount of variance explained by the first component, the first two, the first three and so on. It is easy to notice that most of the variance of the data is contained in the first few components: with only 8 dimensions, more that $90 \%$ of the variance is explained, this confirming the well-known compression capabilities of the PCA technique.

\section{RESULTS}

We focused our attention at the interface between polymer and resin. In our setup, an upright camera coupled with a $20 \times$ objective allows to easily locate features on the sample.

The cross-cut slice exhibit regions where adhesion with the glass substrate is good, the specimen looks planar and multiple folding is not present; materials interface can be easily found by looking at the different color of the two parts. AFM measurements (Fig. 2a) show two different morphologies: the first one (left part of the image) exhibits round reflex features of size ranging from few hundred nanometers to some micrometers, with and peak to valley height of $20-60 \mathrm{~nm}$ : in this region RMS roughness is $\sim 20 \mathrm{~nm}$. The right part of the sample has a flat background with RMS roughness of $5 \mathrm{~nm}$ and convex features of lateral dimension about half a micrometer and height of the order of 50 

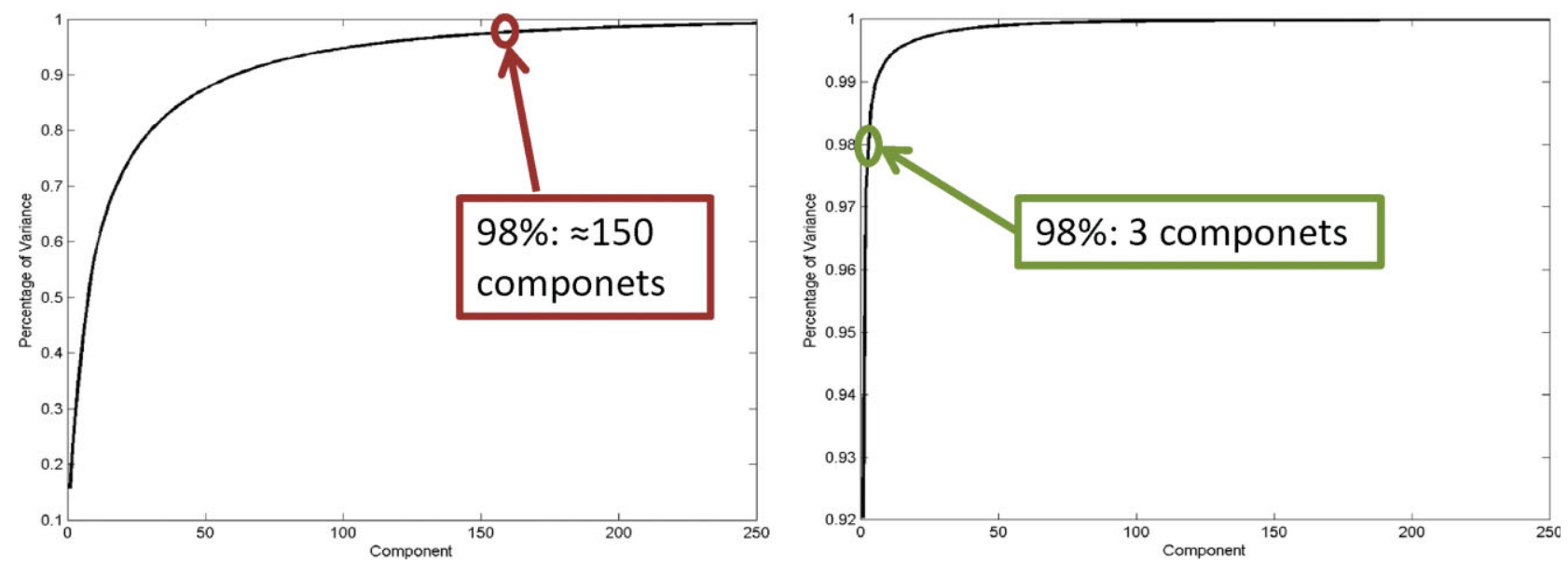

Fig. 3. Convergence of PCA as a function of number of components, in case of Phase (left) and Frequency (right) signals.[Color figure can be viewed in the online issue, which is available at wileyonlinelibrary.com.]

$\mathrm{nm}$. These two parts are recognized as resin and polymer respectively: round features in the first part can be therefore interpreted as gas bubbles produced during solidification of the components. Between the two parts a sharp interface with a size of roughly 100-200 $\mathrm{nm}$ can be identified.

On the basis of these considerations, we made our investigations on an interface region of $5 \times 5 \mathrm{~m}^{2}$, as indicated in Figure 2.

Region shown in Figure $2 \mathrm{~b}$ has been selected as a representative part of the interface for dynamic spectroscopy study, as all the structures previously described are present. Figures $2 \mathrm{c}-2 \mathrm{f}$ show the interaction part of spectroscopy data acquired in position 1 (black curves) and 2 (red curves) of Figure 2b. Amplitude data is taken as a reference for all curves: approach phase ends when it reduces to a fraction of the free oscillation amplitude (flat part of the curve on the right side): in this work we have selected $40 \%$ (trigger point), giving us access to regimes usually difficult to be explored in imaging mode due to possible tip damage; setpoint used for AM imaging is also shown for comparison. The misalignment of the curves $(\Delta H t$ in Fig. 2c) account for the topography of the sample at the end of the curve: this information is subsequently filtered out by pairing all the curves on the left end, correspondingly to trigger point.

All curves have common features that can be highlighted from Figure 2:

1. Very far from the trigger point, the cantilever does not sense any interaction, so all curves are flat, giving no valuable correlation between different curves on each channel and among different channels: eigenvalues of covariance matrix in this part are negligible and do contribute to PCA analysis.

2. Approach and retract part of each spectroscopy data series in general do not overlap completely in the interaction region, but still look very similar. This seems to indicate the effect of plastic behavior on the sample, as expected. Data series at different positions may have very different behavior as a consequence of changes in interaction: some of the channels as frequency and second harmonic seem have particularly high sensitivity to the position of the specimen (e.g., slope in frequency signal in pos 1 and 2): this is spatial information is expected to give the most important contribution to PCA maps, allowing to highlight features in first components of resulting maps.

3. Curves at have response with peculiar tip separation dependence (i.e., average load during each oscillation cycle) with consistent changes at different position. For instance, second harmonic distortion exhaust its main contribution $50 \mathrm{~nm}$ above the Amplitude trigger point in position 2 , while same channel in position 1 still have high sensitivity. Opposite behavior is found on frequency channel. More, amplitude channel on position 2 shows a flexure point at the very beginning of interaction, above imaging setpoint. This behavior is indication of cantilever instability induced by interaction, taking place in a completely different interaction regime, that, as well as other features, contains information on the interaction.

Extracting more information is expected to give useful prediction on the load regime and on the techniques to be used on different part of the sample.

PCA algorithm requires a variable number of components, depending on the channel, for the convergence above the threshold, here 98\%. Figure 3 shows the number of components for frequency and phase shifts channels. Insets contain the number of components to reach convergence. In case of frequency, two components contain all the relevant information, while for phase almost 100 are necessary, as expected from previous considerations on separate spectroscopy curves.

The first principal component on each spectroscopy channel is shown in Figure 4: maps (a), (b), (c), and (d) show results on amplitude, second harmonic, phase and frequency, explaining $82 \%, 65 \%, 15 \%, 92 \%$ of variance (information), respectively. Figure $4 \mathrm{e}$ is the first component (92\% of variance) of PCA on data from all components. Direct comparison of the letter with 

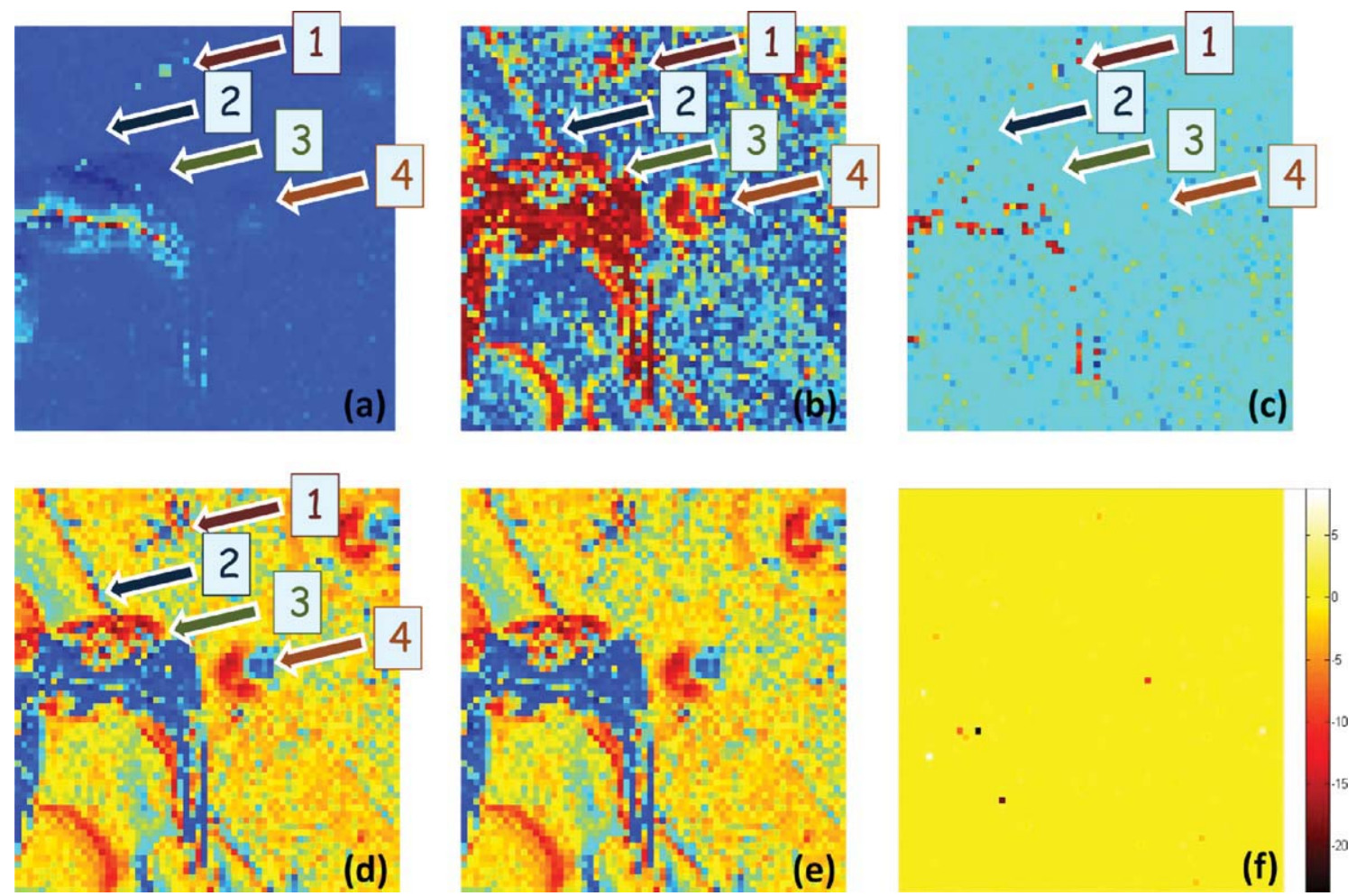

Fig. 4. First component of PCA analysis on dynamic spectroscopy map: Amplitude (a), Second Harmonic (b), Phase (c), frequency (d), and all (e) channels are shown. Map (f) is the difference between (d) and (e): color bar scale is part per thousand.

Figure $4 \mathrm{~d}$ indicate that the most important contribution comes from frequency channel, since differences deriving from other contribution stays in the few percent range (Fig. 4f).

All data in Figure 4 is acquired in the square $5-\mu \mathrm{m}$ region shown in Figure 2b: some of the features in the image, such as the three bubbles on the resin side or the tree convex features on the polymer side, can be found in most of the maps, with the exclusion of phase one where just some contrast on one of them (Arrow 1) can be located. On some of the maps (frequency and second harmonic channels) can also be clearly recognized interface between the two materials (Arrow 2), providing much lower contrast on Amplitude channel. Surprisingly, a new feature, not visible in topography data, appears in the left part of all the maps [Arrow 3, see map (b)] whose substructure are differently highlighted from different channels: this suggest a complex inner structure that can be evaluated by our method. Three different regions can be recognized comparing Figure $4 \mathrm{~b}$ to the others: first, all the part in map $4 \mathrm{~b}$ has similar values (is red in the false color map), as an indication of similar harmonic distortion (on the second component) in spectroscopy curves. Other behavior is found on frequency channel, where top part (red) and bottom part (blue) give opposite contribution to frequency shift. Finally, a central thin feature can be found: there a high contrast can be noticed on phase and amplitude signals, while it is invisible on second harmonic; here also very small contribution (yellow, average value) even to the frequency signal is found.
Similar behavior is found on other structures, also visible in topography, as the one indicated by Arrow 4, and magnified in Figure 5.

Figures $5 \mathrm{a}-5 \mathrm{~d}$ are magnified details of Figures $4 \mathrm{a}-4 \mathrm{~d}$, while Figures 5e-5h show correspondent spectroscopy curves, acquired in the two positions pointed by arrows in Figure 5a. All the channels have similar response in both position at low interactions (between 120 and $150 \mathrm{~nm}$ ): at higher interaction it is possible to se huge change on frequency shift (opposite sign) and second harmonic spectroscopy curve (h) have a second maximum (higher) in one of the two positions, giving indication on different compositional or structural properties.

\section{DISCUSSION}

In composite materials, structural and compositional information determinate local properties, that can be directly measured by mean of atomic force microscope. Discontinuities at surface affect cantilever motion, generating correlated changes in its dynamic parameters: this correlation contains relevant information on the interaction potential detectable by means of force spectroscopy experiments. Dynamic force spectroscopy experiment can provide an extensive data set, allowing specimen testing under different frequency and load condition at once. It is therefore a powerful method to test material properties. More, structural changes do not necessary bind to topography features, hence the examination of surface morphology do not directly provide compositional information and can be incomplete or misleading in analyzing multiphase or 

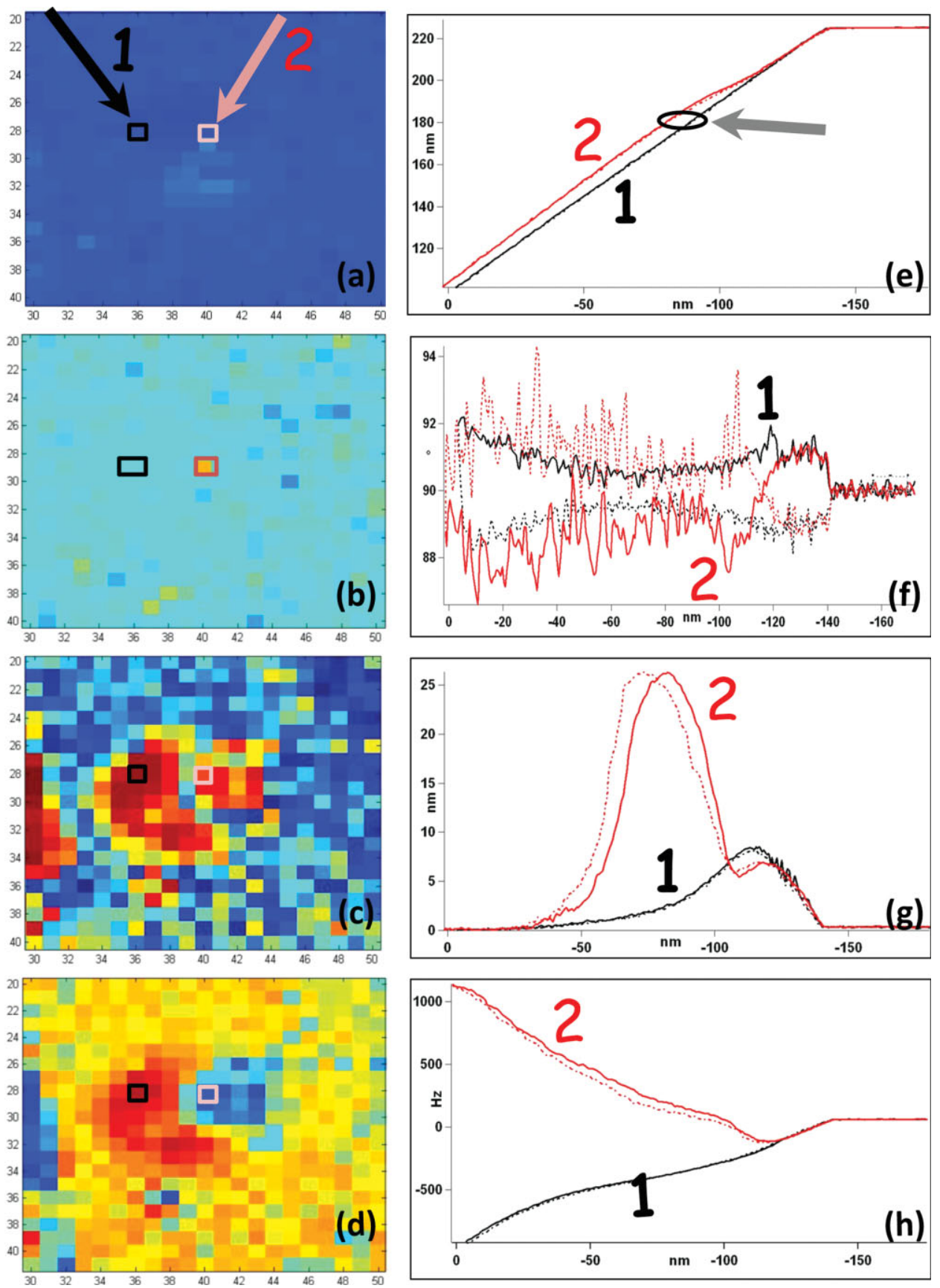

Fig. 5. Magnification one of the features highlighted in previous figure (Arrow 4). Red and black curves in subpanel (e-h) are amplitude, phase, second harmonic amplitude, and frequency channels of spectroscopy curves acquired in two point indicted by red and black markers (respectively) on maps (a-c), showing in details first compo-

nent of PCA analysis on those channels. Gray arrow shows the imaging setpoint used for topography. As before continuous lines indicates approach curves, while dotted lines are for retract curves. [Color figure can be viewed in the online issue, which is available at wileyonlinelibrary.com.]

textured specimen. From here the necessity of a method addressing both issues separately.

In this article, we have shown a different strategy to address the problem of compositional recognition, introducing an intermediate step to localize features, simply taking into consideration cantilever kinematics: as this quick screening does not require detailed modeling of the contact potential, can therefore be used routinely to identify part of the sample where a more detailed investigation can be addressed.

This analysis addresses three main goals:

1. Hidden features can be highlighted and recognized. This method does not take into account topography information that is filtered out from the data: maps and images are regarded as independent. As result 
it allowed recognition of some hidden structures (possibly below the surface), invisible in topography image (Fig. 4).

2. Different channels are analyzed to give complete information. The effect of physical interaction on several oscillation parameters is analyzed in parallel, giving a global picture on different response on the sample. This allows easily locating different ROIs before a detailed and time consuming spectroscopy analysis. More, this method can be followed by segmentation step to classify and reduce the number of curves to be analyzed within similar or uniform region of the sample. Noise and redundancies are filtered out by the algorithm itself.

3. Relative weight of different channels is highlighted. This gives indication on the channels and measurements regime (e.g., average load) that can be used to enhance compositional or structural sensitivity were more information can be collected, within each part of the sample. Then a reduced number of optimized experiments can be performed on a reduced number of experimental points for model comparison.

Finally, it should be reminded that PCA algorithm just looks at mathematical correlations in parallel data vectors and project information into the high correlation directions of covariance matrix. In this way, interpretation of time series in terms of physical parameters is lost, to give a compact and easily valuable picture. In any case, this method can provide non destructive additional information on sample properties and homogeneity, can therefore be regarded as a quick and easy preliminary step to enhance and relieve detailed analysis and modeling.

\section{ACKNOWLEDGMENTS}

The authors acknowledge G. Bertoni, M. Malerba, and D. Fragouli for useful discussions and help in sample preparation. They also thank Dr. Jason Bemis for assistance with Igor Pro code.

\section{REFERENCES}

Butt HJ, Cappella B, Kappl M. 2005. Force measurements with the atomic force microscope: Technique, interpretation and applications. Surf Sci Rep 59:1-152.

Cappella B, Dietler G. 1999. Force-distance curves by atomic force microscopy. Surf Sci Rep 34:1-104.
Derjaguin BV, Muller VM, Toporov YP. 1975. Effect of contact deformations on the adhesion of particles. J Colloid Interface Sci 53:314325 .

Durig U. 1999. Relations between interaction force and frequency shift in large-amplitude dynamic force microscopy. Appl Phys Lett 75:433-435.

Durig U. 2000. Extracting interaction forces and complementary observables in dynamic probe microscopy. Appl Phys Lett 76:12031205

Garcia R, Perez R. 2002. Dynamic atomic force microscopy methods. Surf Sci Rep 47:197-301.

Giessibl FJ. 1997. Forces and frequency shifts in atomic-resolution dynamic force microscopy. Phys Rev B 56:16010-16015.

Hertz H. 1889. Über die Berührung fester elastischer Körper. J Reine Angew Math 92:156-171.

Holscher H. 2006. Quantitative measurement of tip-sample interactions in amplitude modulation atomic force microscopy. Appl Phys Lett 89:123109 1-3.

Holscher H. 2008. Theory of phase-modulation atomic force microscopy with constant-oscillation amplitude. J Appl Phys 103: 064317.

Holscher H, Schwarz UD, Zworner O, Wiesendanger R. 1998. Consequences of the stick-slip movement for the scanning force microscopy imaging of graphite. Phys Rev B 57:2477-2481.

$\mathrm{Hu}$ S, Raman A. 2008. Analytical formulas and scaling laws for peak interaction forces in dynamic atomic force microscopy. Nanotechnology 19:375704 1-11.

Jamitzky F, Stark M, Bunk W, Heckl WM, Stark RW. 2006. Chaos in dynamic atomic force microscopy. Nanotechnology 17:S213-S220.

Jesse S, Kalinin SV. 2009. Principal component and spatial correlation analysis of spectroscopic-imaging data in scanning probe microscopy. Nanotechnology 20:085714 1-7.

Jesse S, Kalinin SV, Proksch R, Baddorf AP, Rodriguez BJ. 2008. The band excitation method in scanning probe microscopy for rapid mapping of energy dissipation on the nanoscale. Nanotechnology 18:435503 1-8.

Johnson KL, Kendall K, Roberts AD. 1971. Surface energy and the contact of elastic solids. Proc R Soc London A 324:301-313.

Jollife IT. 1986. Principal component analysis. New York: Springer Verlag.

Lee M, Jhe W. 2006. General theory of amplitude-modulation atomic force microscopy. Phys Rev Lett 97:036104 1-4.

Magonov SN, Elings V, Papkov VS. 1997. AFM study of thermotropic structural transitions in poly(diethylsiloxane). Polymer 38:297307.

Sader JE, Jarvis SP. 2004. Accurate formulas for interaction force and energy in frequency modulation force spectroscopy. Appl Phys Lett 84:1801-1803.

Sader JE, Uchihashi T, Higgins MJ, Farrell A, Nakayama Y, Jarvis SP. 2005. Quantitative force measurements using frequency modulation atomic force microscopy-theoretical foundations. Nanotechnology 16:S94-S101.

Sugawara Y, Kobayashi N, Kawakami M, Li YJ, Naitoh Y, Kageshima M. 2007. Elimination of instabilities in phase shift curves in phasemodulation atomic force microscopy in constant-amplitude mode. Appl Phys Lett 90:194104 1-3.

Unertl WN. 1999. Implications of contact mechanics models for mechanical properties measurements using scanning force microscopy. J Vac Sci Technol A 17:1779-1786. 